\title{
Biomarker: Trolox equivalent antioxidant capacity and telomere length of Thai elderly people with frailty
}

Jetsada Ruangsuriya1', Penprapa Siviroj2* , Pisittawoot Ayood², Krongporn Ongprasert², Navakoon Kaewtunjai ${ }^{1}$, Somdet Srichairatanakool', Wirote Tuntiwechapikul', Jiraporn Chittrakul ${ }^{2}$

'Department of Biochemistry, Faculty of Medicine, Chiang Mai University, Chiang Mai, Thailand, 50200, ²Department of Community Medicine, Faculty of Medicine, Chiang Mai University, Chiang Mai, Thailand, 50200

\section{ABSTRACT}

Introduction: Thailand has a rapidly aging population; assessing frailty earlier with biochemical markers could identify many adverse outcomes such as disability, hospitalization, and death. We aimed to examine the correlation between Trolox equivalent antioxidant capacity (TEAC), thiobarbituric acid reactive substances (TBARS), and telomere length and frailty for elderly people in Northern Thailand.

Methods: A cross-sectional study was performed between May 2017 and March 2018 with a total of 350 elderly aged 60 and older for frailty phenotype assessment by five frailty criteria including unintentional weight loss, exhaustion, slowness, low physical activities, and grip strength weakness. Twenty-eight subjects in both the frailty and non-frailty groups were analyzed for basic clinical parameters, including plasma TEAC, TBARS, and telomere length.

Results: Alanine aminotransferase activity, albumin concentration, cholesterol level, and telomere length were significantly low in the frailty group. The albumin level, TEAC, and telomere length were significantly higher between the ages of 60 and 75 years compared to those with non-frailty over 75 years of age. Likewise, albumin and cholesterol levels were significantly higher in the frailty group aged 60-75 years. Albumin concentration, cholesterol level, TEAC, and telomere length were significantly higher in the non-frailty group when compared to the frailty group aged 60-75 years, but no significant difference was found among these biochemical parameters of frailty and non-frailty whose age was above 75 years.

Conclusion: The reduction of albumin concentration, cholesterol level, TEAC, and telomere length supports the underlying mechanism of frailty screened by the frailty phenotype tool in a specific age range. However, further analyses with multi-parameters must be validated before the application in clinical diagnosis for frailty.

Key words: Frailty; elderly; biomarker; Trolox equivalent antioxidant capacity; telomere length

*Corresponding author: Penprapa Siviroj, Department of Community Medicine, Faculty of Medicine, Chiang Mai University Chiang Mai, 110 Inthavaroros, Sriphum, Muang, Chiang Mai, 50200, Thailand. Tel: +66 53 935472-4, Fax: 6653 935476,

E-mail: psiviroj@gmail.com

Submitted: 21 May 2019/Accepted: 30 September 2019

DOI: https://doi.org/10.17532/jhsci.2019.737

UNIVERSITY OF SARAJEVO FACULTY OF HEALTH STUDIES

\section{INTRODUCTION}

One great benefit from medical technology and modern medicine is the longevity of the humankind, which unavoidably is turning into an aging society currently emerging around the world. The United Nations expects the world elderly population with age over 60 
to increase by $56 \%$ with a total number of 2.1 billion by 2050 . The shift of population to an aging society affects world economic and sociology concerns in many aspects. One major concern regarding this shift is the health care of the elders, which has become the priority in many countries (1). Another important factor affecting the health condition of the elders is frailty. To assess frailty, the gold standard protocol is a phenotypic evaluation, in which the three out of the five following positive determinants are found in an individual; (a) unintentional weight loss, (b) exhaustion, (c) slow walking speed, (d) loss of physical activities, and (e) low grip strength (2). Recently, no specific biochemical marker could be applied to differentiate the frailty and non-frailty groups; however, chronic inflammation and its related markers have been reported as a major determinant of the frailty syndrome in elders (3).

Supposing that the routine blood biochemistry could determine the frailty and non-frailty category, it would be of benefit for the elders during their regular health checkups. In general, the results from routine blood biochemistry primarily indicate certain organ defects. For example, liver function could be determined by the rise in activities of these enzymes in the blood; aspartate aminotransferase (AST), alanine aminotransferase (ALT), and alkaline phosphatase (ALP) (4). In contrast, the level of urea and albumin is lower than the normal range (5). Kidney function could be assessed by an increasing level of creatinine in either blood or urine. Likewise, the significant rise in the level of triglycerides (TG) and total cholesterol increase the risk of cardiovascular defects (6). The gaps between frailty and non-frailty in terms of these biochemical parameters have been doubtful. This is because frailty results from multidimensional players such as genetic, biological, physical, psychological, social, and environmental factors, although inflammation and oxidative stress play an important role in the development of fragile states $(7,8)$. In addition to basic routine blood biochemistry, the promising factors supporting frailty have been inflammation producing promiscuous oxidants and antioxidants before, during, and after the process. In normal cases, the balance between the degree of Trolox equivalent antioxidant capacity (TEAC) and the level of an oxidant such as reactive oxygen species (ROS) has been tightly maintained.
The imbalance of these parameters potentially poses certain abnormalities.

TEAC is the cumulative activity of the body to neutralize oxidants, such as ROS, produced during cellular metabolism. The higher the level of TEAC indicates the greater the capacity to protect cellular damage from ROS. The levels of TEAC were found to be significantly higher in normal volunteers than that in certain diseases such as inflammatory bowel disease (9) and diabetes mellitus (10).

In contrast, an oxidant like ROS can damage cellular structures leading to cellular dysfunction. The measurement of ROS in the sample is difficult due to its molecular instability. Alternatively, one of the results from ROS is lipid peroxidation product so-called malondialdehyde (MDA) measured by thiobarbituric acid-reactive substances (TBARS) indicating the oxidation state of the cells in the body. Despite nonspecificity to the MDA, TBARS is widely accepted as a common method to determine the MDA in cells, tissues, and body fluids (11). For example, TBARS has been used as the biochemical marker for oxidative stress as its level is significantly increased in the involved diseases such as cardiovascular disease (12) and cancer (13).

Shortened telomeres, an intracellular marker, indicate cellular senescence. Telomeres are recognized as special structures at the ends of the chromosomal DNA with the function of stabilizing chromosomes. Telomere shortening with increasing age and under certain pathological conditions has been well known (14). Even though the telomere length marker is not specific for diseases, it has been used as a biochemical marker for cardiovascular disease (15).

It is because frailty potentially worsens the health conditions and leads to disability and much worse to fatality in combination with simple ailments (16). In the present study, the aim was to find the relationship between biochemical markers, including ALT activity, albumin level, total cholesterol, TEAC, TBARS, and telomere length in the elders identified in the frailty or non-frailty group. Ultimately, the findings could be potentially applied to the elders who come into the hospital for a regular checkup by increasing awareness of ailments if they are tending to be in a frailty classification. 


\section{METHODS}

\section{Subject recruitment}

Three-hundred-fifty elderly people with the age over 60 years living in the Pasang District, Lamphun Province, Thailand, were recruited for frailty and non-frailty determination using a physical phenotype method (16). Briefly, five criteria for frailty determination included unintentional weight loss, exhaustion, slowness in a distance of 15 -feet, low activities, and weakness of grip strength. Frailty, pre-frailty, and nonfrailty were classified when the above criteria were positive for any $3-5,1-2$, and none of them, respectively. Twenty-eight subjects in each group of the frailty group and the non-frailty group were recruited for blood sample collection. This research was approved by the Research Ethics Committee for Human Research of Faculty of Medicine, Chiang Mai University under the approval number of 415/2016.

\section{Blood sample collection}

Five milliliters of fasting venous blood were drawn into each heparin-coated tube. The tubes were kept cold on ice and subjected to centrifugation for 5 min to separate plasma and the blood cells for further analyses (17). The plasma was kept at $-20^{\circ} \mathrm{C}$ for routine clinical chemistry analyses, TEAC, and TBARS. The white blood cells were further processed for DNA extraction and telomere length determination.

\section{Determination of basic clinical laboratory parameters in plasma}

Urea, creatinine, liver enzyme activities, AST, ALT, and ALP, total cholesterol, TG, and albumin were determined using the automating machine (Randox, RX Daytona, UK).

\section{Determination of TEAC}

The ATBS solution was prepared according to the method described elsewhere (18). Ten microliters $(\mu \mathrm{L})$ of plasma were mixed with the ATBS solution. The reaction was allowed for $6 \mathrm{~min}$ and the absorbance at $734 \mathrm{~nm}$ was determined. The TEAC in each plasma sample was calculated using Trolox standard curve and the TEAC was reported as millimolar Trolox equivalent (mM).

\section{Determination of TBARS by thiobarbituric acid assay}

The assay for TBARS determination was modified from Kamal et al. (19) by which $50 \mu \mathrm{L}$ of plasma was deproteinized with butylated hydroxytoluene in $10 \%$ trichloroacetic acid. The solution was mixed with $0.6 \%$ thiobarbituric acid in $0.44 \mathrm{M}$ phosphoric acid. The pink color developed after a $30 \mathrm{~min}$ incubation at $90^{\circ} \mathrm{C}$ and the absorbance was measured at $532 \mathrm{~nm}$. The TBARS level was calculated using $1,1,3,3$ tetramethoxypropane standard curve and reported in micromolar $(\mu \mathrm{M})$.

\section{Determination of telomere length}

Total DNA was extracted from white blood cells using a GF-1 Blood DNA Extraction Kit (Vivantis, Malaysia). Twenty nanograms (ng) of the extracted DNA were subjected to telomere length determination following the method described elsewhere by monochrome multiplex quantitative polymerase chain reaction (20). The telomere length was reported as a ratio of telomere repeat copy number to single-copy gene copy number (T/S ratio).

\section{Statistical analysis}

Demographics, medical history, and clinical parameters were tested by the Mann-Whitney $U$-test and Fisher's exact test. Spearman correlation coefficients were calculated for the association between biological marker parameters.

\section{RESULTS}

Statistical analyses showed that means of age, ALT activity, total cholesterol, albumin level, and telomere length were significantly different between the non-frailty and frailty groups. The average age of non-frailty volunteers was statistically lower than that of frailty subjects. In contrast, the average values of ALT activity, albumin level, total cholesterol, and telomere length in non-frailty volunteers were significantly higher than those in frailty subjects. On the other hand, the body mass index, sex, illness status (diabetes and cardiovascular diseases), routine clinical biochemical test (creatinine, urea, AST, and TG), Trolox equivalent, and TBARS were not significantly different between the non-frailty and frailty groups (Table 1). 
TABLE 1. Mean \pm standard deviation of clinical parameters of older adults in non-frailty and frailty groups

\begin{tabular}{lccc}
\hline Parameters & Non-frailty $(\mathrm{n}=28)$ & Frailty $(\mathrm{n}=28)$ & $p$-value \\
\hline Age (years) & $67.79 \pm 6.70$ & $75.43 \pm 8.34$ & $<0.001$ \\
Sex (female), $\mathrm{n}(\%)$ & $20(71.4)$ & $6(27.3)$ & 0.758 \\
BMI $\left(\mathrm{kg} / \mathrm{m}^{2}\right)$ & $22.76 \pm 3.02$ & $23.64 \pm 4.07$ & 0.356 \\
Hypertension, $\mathrm{n}(\%)$ & $10(55.6)$ & $20(71.4)$ & 0.016 \\
Diabetes, $\mathrm{n}(\%)$ & $3(12.0)$ & $8(40.0)$ & 0.178 \\
Cardiovascular diseases, $\mathrm{n}(\%)$ & $2(7.7)$ & $0(0.0)$ & 0.491 \\
Creatinine $(\mathrm{mg} / \mathrm{dL})[0.6-1.1 \mathrm{mg} / \mathrm{dL}]$ & $1.085 \pm 0.346$ & $1.075 \pm 0.325$ & 0.961 \\
Urea $(\mathrm{mg} / \mathrm{dL})[5-25 \mathrm{mg} / \mathrm{dL}]$ & $13.348 \pm 4.730$ & $13.354 \pm 6.265$ & 0.502 \\
AST $(\mathrm{U} / \mathrm{L})[0-37 \mathrm{U} / \mathrm{L}]$ & $24.643 \pm 6.547$ & $22.965 \pm 5.782$ & 0.355 \\
ALT $(\mathrm{U} / \mathrm{L})[0-40 \mathrm{U} / \mathrm{L}]$ & $10.030 \pm 4.623$ & $7.085 \pm 2.780$ & 0.011 \\
ALP $(\mathrm{U} / \mathrm{L})[30-120 \mathrm{U} / \mathrm{L}]$ & $69.479 \pm 19.422$ & $65.834 \pm 16.635$ & 0.523 \\
Albumin $(\mathrm{g} / \mathrm{dL})[3.5-5.0 \mathrm{~g} / \mathrm{dL}]$ & $5.411 \pm 0.174$ & $4.693 \pm 0.580$ & $<0.001$ \\
Total cholesterol (mg/dL) $[0-150 \mathrm{mg} / \mathrm{dL}]$ & $204.893 \pm 31.231$ & $171.893 \pm 36.738$ & 0.001 \\
Triglyceride $(\mathrm{mg} / \mathrm{dL})[0-150 \mathrm{mg} / \mathrm{dL}]$ & $113.321 \pm 52.374$ & $94.393 \pm 38.272$ & 0.134 \\
Trolox equivalent $(\mathrm{mM})$ & $1.819 \pm 0.067$ & $1.791 \pm 0.091$ & 0.190 \\
TBARS $(\mu \mathrm{M})$ & $3.877 \pm 1.463$ & $3.370 \pm 0.955$ & 0.132 \\
Telomere length (T/S ratio) & $0.505 \pm 0.264$ & $0.289 \pm 0.174$ & 0.001 \\
\hline
\end{tabular}

The values show either mean \pm standard deviation or the number of subjects who are positive/ the number of subjects who are negative; the values written in [] are normal reference ranges and NS stands for not significant difference between non-frailty and frailty groups. ALT: Alanine aminotransferase; ALP: Alkaline phosphatase; AST: Aspartate aminotransferase; TBARS: Thiobarbituric acid-reactive substances; BMI: Body mass index

Because the age was significantly different between non-frailty and frailty groups, it was wondered whether clinical parameters were correlated with age. It was found that significantly negative correlations were observed between the age and the activity of ALT $(r=-0.402, p=0.002)$, the level of albumin $(\mathrm{r}=-0.588, \mathrm{p}<0.001)$, the total cholesterol level $(\mathrm{r}=-0.453, \mathrm{p}<0.001)$, and the telomere length $(\mathrm{r}=-0.460, \mathrm{p}<0.001)$. In addition, the correlation among clinical parameters was also analyzed within all participants. Significant positive correlations were found between the level of urea and creatinine, ALT activity and albumin level, ALT and AST activities, ALP activity and TBARS level, total cholesterol and albumin level, total cholesterol and TBARS, and total cholesterol and telomere length (Table 2).

Twenty-eight volunteers in either the non-frailty or frailty groups were further divided by age, which were between 60 and 75 years and above 75 years. The level of albumin, total cholesterol, TEAC, TBARS, and the telomere length was significantly higher in the non-frailty group with the ages of 60-75 years when compared with those in the nonfrailty group with the ages $>75$ years. Likewise, the
TABLE 2. Correlation between clinical parameters measured in 56 participants using spearman rank correlation coefficient

\begin{tabular}{lcc}
\hline Correlated parameters & Correlation coefficient & $p$-value \\
\hline Urea & & \\
Creatinine & 0.690 & $<0.001^{* *}$ \\
$\quad$ Albumin & -0.273 & $0.042^{*}$ \\
ALT & & \\
Albumin & 0.445 & $<0.001^{* *}$ \\
AST & 0.434 & $<0.001^{\text {** }}$ \\
ALP & & \\
TBARS & 0.332 & $0.012^{*}$ \\
Total cholesterol & & \\
Albumin & 0.465 & $<0.001^{* *}$ \\
TBARS & 0.279 & $0.037^{*}$ \\
Telomere length & 0.276 & $0.040^{*}$ \\
\hline
\end{tabular}

${ }^{*}$ Significant (two-tailed) at $0.05 ;$; ${ }^{*}$ Significant (two-tailed) at 0.01 . ALT: Alanine aminotransferase; ALP: Alkaline phosphatase; AST: Aspartate aminotransferase; TBARS: Thiobarbituric acid reactive substances

same parameter levels were significantly higher in the frailty groups. In addition, the levels of total cholesterol, albumin, Trolox equivalent, and telomere length were significantly higher in the non-frailty 
group compared to the frailty group with the ages of 60-75 years (Table 3 ). There was no any parameter showing differences between the non-frailty and frailty groups with the ages above 75 years.

\section{DISCUSSION}

Five parameters; age, ALT activity, albumin level, total cholesterol, and telomere length, were significantly different between the non-frailty and frailty groups. Albumin level, total cholesterol concentration, and telomere length tended to be associated with frailty. Among those three parameters, telomere length is the most evident to be associated with aging.

The telomere length of the frailty groups was significantly lower than that of the non-frailty group. It was consistent with numerous reports indicating the shortening of telomere had a strongly negative association with the aging process rather than frailty phenotypes. Woo et al. suggested that the telomere shortening was found only in men, but not in women as well as no significant correlation between frailty index and telomere length (21). Likewise, the ESTHER cohort studies reported that the telomere length was inversely related only with age, but not with the frailty score (22). In contrast, the telomere length was not different between the frailty and the non-frailty groups of Chinese whose age was 65 or older (23). Recently, the meta-analysis of telomere length and frailty statuses indicated no association between these two factors (24). Together, it is still unclear whether the use of telomere alone as a biochemical marker for frailty is sufficient for frailty identification.

After analysis of biochemical parameters according to age group, it was shown that the frailty group had significantly lower levels of total cholesterol, albumin, and TEAC. These results were consistent with the report that the highest risk of death, indicating frailty, within 4.5 years was significantly found in subjects of which the total blood cholesterol and albumin concentrations were lower than $160 \mathrm{mg} / \mathrm{dL}$ and $38 \mathrm{~g} / \mathrm{L}$, respectively (25). Likewise, the subjects who had total blood cholesterol and albumin lower than $5.2 \mathrm{mM}$ and $43 \mathrm{~g} / \mathrm{L}$, respectively, showed a significant reduction in functional performance within 3 years, indicating frailty (26). One explanation is that most of the older population tended to eat less compared with other ages, and it adversely resulted in poor nutrition which leads to oxidative stress and finally to frailty (27). Ongoing malnutrition of frailty subjects led to death indicated by the low albumin level, but elevated C-reactive protein (CRP), as well as urea levels, was also noted. Even though those levels were in the normal ranges, a statistical significance was found between the frailty group who had a long life and the deceased group (28). Several reports have claimed specific biochemical markers for frailty. For example, an increase in serum 8-hydroxy-2-deoxyguanosine was observed in the frailty group with a positive correlation with the frailty score (29). Moreover, the study with proteomic techniques suggested at least seven glycoproteins expressing differently between frailty and non-frailty groups (30). The latter evidence might give the researcher a clue not to focus on only one biomarker for frailty, requiring advanced techniques as well as analytical tools for frailty marker diagnosis, and further studies.

Our findings regarding ALT activity indicated a significant reduction of the enzyme activity in the frailty group when the data were analyzed regardless

TABLE 3. Levels of total cholesterol, albumin, Trolox equivalent, and telomere length in subjects with non-frailty compared to frailty in the group's ages $60-75$ and $>75$ years

\begin{tabular}{lccccc}
\hline Parameters & \multicolumn{2}{c}{ Non-frailty $(\mathrm{n}=28)$} & & \multicolumn{2}{c}{ Frailty $(\mathrm{n}=28)$} \\
\cline { 2 - 3 } \cline { 5 - 6 } \cline { 5 - 6 } & Age 60-75 years $(\mathrm{n}=23)$ & Age $>75$ years $(\mathrm{n}=5)$ & & Age 60-75 years $(\mathrm{n}=17)$ & Age $>75$ years $(\mathrm{n}=11)$ \\
\hline Albumin $(\mathrm{g} / \mathrm{dL})$ & $4.153 \pm 0.173^{*}$ & $3.978 \pm 0.090$ & & $3.912 \pm 0.189^{\#, \$}$ & $3.612 \pm 0.429$ \\
Total cholesterol $(\mathrm{mg} / \mathrm{dL})$ & $206.478 \pm 28.279$ & $197.600 \pm 45.873$ & & $182.471 \pm 40.221^{\#, @}$ & $155.546 \pm 23.906$ \\
Trolox equivalent $(\mathrm{mM})$ & $1.832 \pm 0.064^{*}$ & $1.757 \pm 0.043$ & & $1.775 \pm 0.072^{\$}$ & $1.815 \pm 0.115$ \\
Telomere length $(\mathrm{kb})$ & $5.594 \pm 0.837^{*}$ & $4.567 \pm 0.547$ & & $4.752 \pm 0.601^{\$}$ & $4.601 \pm 0.561$ \\
\hline
\end{tabular}

The values show mean \pm standard deviation. ** ,and @indicate the significant at $p<0.05$ when compared with non-frailty ages $>75$ years,

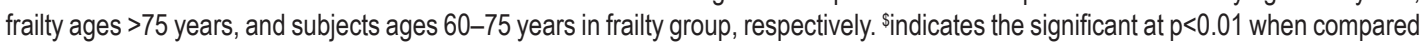
with and subjects ages $60-75$ years in frailty group 
of the age sub-classification. This study indicated that the ALT activity in the frailty group was in the normal range with lower activity in the non-frailty group. The result was supported by the finding in middle-aged adults that subjects with low normal ALT activity had an increased risk of long-term mortality (31).

The TEAC found in the frailty group was significantly low when the data were analyzed within the corresponding age group, whereas the level of oxidant reported in TBARS did not differ between non-frailty and frailty. The insignificant difference of TBARS in between non-frailty and frailty might be a result of the nonspecificity of TBARS to MDA as mentioned earlier (32). However, this finding indicated an imbalance between the oxidant and antioxidant systems, which lead to oxidative stress and frailty as supported by various evidence. Frailty has been claimed to be associated with inflammation indicated with the expression of pro-inflammatory cytokines resulting in oxidative stress. In patients with sarcopenia, frailty was associated with an increase in tumor necrosis factor- $\alpha$, interleukins, and CRP indicating the oxidative stress measured by the level of ROS (33). The significant increase in TBARS was observed in frailty subjects and had a strong correlation between these two factors (34). Recently, the meta-analysis of frailty and oxidative stress revealed an association between oxidative stress and frailty. However, the association between the levels of oxidant and antioxidant capacity remained inconclusive with the trends of increasing in oxidant and decreasing in antioxidants (35).

Another point of interest was that when all biochemical parameters of the subjects who were above 75 years between the non-frailty and frailty groups, none of the parameters were significantly different. This might be due to the number of subjects between those groups. It was consistent with Collerton et al. report that there was no association of clinical parameters in the very old subject group whose age was 85 or older (36).

Taken together, our results added the information that the frailty biomarker could not be only a specific one. The use of basic routine biochemical markers to diagnose the frailty could be possible only in the case of multiple analyses of multiple parameters.
Therefore, further studies must be done to validate further clinical applications.

\section{CONCLUSION}

This study supported the frailty phenotype-screening tools that there were significantly low levels of biochemical parameters in frailty subjects. With regard to routine biochemical measurement, we found a reduction in albumin concentration and total cholesterol levels involved in frailty. TEAC could also be used additionally in the case that the subjects were between 60 and 75 years. While the telomere length seemed to be efficient as a frailty marker, it was rather an aging marker than frailty. Further analyses with multi-parameters in a greater number of subjects must be studied to deeply understand the multi-parameter mechanism of frailty.

\section{CONFLICTS OF INTEREST}

The author(s) declared no potential conflicts of interest with respect to the research, authorship, and/or publication of this article.

\section{ACKNOWLEDGMENT}

This research was granted by the Faculty of Medicine, Chiang Mai University, Thailand (BIO2559-04207) and the Chiang Mai University Research Fund (BIO-2558-02903). The researchers are also grateful to all the volunteer staff of a HealthPromoting Hospital in Pasang District, Lamphun Province, Thailand. Much gratitude is offered to all subjects participating in this study.

\section{REFERENCES}

1. Loser C, Fajgenbaum J, Kohli HA, Vilkelyte I. How aging societies may affect global growth prospects. Glob J Emerg Mark Econ 2017;9:38-74. https://doi.org/10.1177/0974910117747770.

2. Buckinx F, Rolland Y, Reginster JY, Ricour C, Petermans J, Bruyère $O$. Burden of frailty in the elderly population: Perspectives for a public health challenge. Arch Public Health 2015;73:19.

https://doi.org/10.1186/s13690-015-0068-X.

3. Chen X, Mao G, Leng SX. Frailty syndrome: An overview. Clin Interv Aging 2014;9:433-41.

4. Dufour DR, Lott JA, Nolte FS, Gretch DR, Koff RS, Seeff LB. Diagnosis and monitoring of hepatic injury. II. Recommendations for use of laboratory tests in screening, diagnosis, and monitoring. Clin Chem 2000;46:2050-68.

5. Nakano M, Worner TM, Lieber CS. Perivenular fibrosis in alcoholic liver injury: Ultrastructure and histologic progression. Gastroenterology 1982;83:777-85. 
6. Wilson PWF, D'Agostino RB, Levy D, Belanger AM, Silbershatz H, Kannel WB. Prediction of coronary heart disease using risk factor categories. Circulation 1998;97:1837-47.

https://doi.org/10.1161/01.cir.97.18.1837.

7. Mulero J, Zafrilla P, Martinez-Cacha A. Oxidative stress, frailty and cognitive decline. J Nutr Health Aging 2011;15:756-60.

https://doi.org/10.1007/s12603-011-0130-5.

8. Viña J, Borras C, Gomez-Cabrera MC. A free radical theory of frailty. Free Radic Biol Med 2018;124:358-63.

https://doi.org/10.1016/j.freeradbiomed.2018.06.028.

9. Koutroubakis IE, Malliaraki N, Dimoulios PD, Karmiris K, Castanas E, Kouroumalis EA. Decreased total and corrected antioxidant capacity in patients with inflammatory bowel disease. Dig Dis Sci 2004;49:1433-7. https://doi.org/10.1023/b: ddas.0000042242.22898.d9.

10. Vessby J, Basu S, Mohsen R, Berne C, Vessby B. Oxidative stress and antioxidant status in Type 1 diabetes mellitus. J Intern Med 2002;251:69-76. https://doi.org/10.1046/j.1365-2796.2002.00927.x.

11. Landau G, Kodali VK, Malhotra JD, Kaufman RJ, Cadenas E, Packer L. Detection of oxidative damage in response to protein misfolding in the endoplasmic reticulum. Methods Enzymol 2013; 526:231-50.

https://doi.org/10.1016/b978-0-12-405883-5.00014-4.

12. Walter MF, Jacob RF, Jeffers B, Ghadanfar MM, Preston GM, Buch J, et al. Serum levels of thiobarbituric acid reactive substances predict cardiovascular events in patients with stable coronary artery disease: A longitudinal analysis of the prevent study. J Am Coll Cardiol 2004;44:1996-2002.

https://doi.org/10.1016/j.jacc.2004.08.029.

13. Senthil K, Aranganathan S, Nalini N. Evidence of oxidative stress in the circulation of ovarian cancer patients. Clin Chim Acta 2004;339:27-32.

https://doi.org/10.1016/j.cccn.2003.08.017.

14. Aviv A. Telomeres and human somatic fitness. J Gerontol A Biol Sci Med Sci 2006;61:871-3.

15. De Meyer T, Rietzschel ER, De Buyzere ML, Van Criekinge W, Bekaert S. Telomere length and cardiovascular aging: The means to the ends? Ageing Res Rev 2011;10:297-303.

https://doi.org/10.1016/j.arr.2010.11.001.

16. Fried LP, Tangen CM, Walston J, Newman AB, Hirsch C, Gottdiener J, et al. Frailty in older adults: Evidence for a phenotype. J Gerontol A Biol Sci Med Sci 2001;56:M146-56. https://doi.org/10.1093/gerona/56.3.m146.

17. Kambayashi $Y$, Binh NT, Asakura HW, Hibino $Y$, Hitomi $Y$, Nakamura H, et al. Efficient assay for total antioxidant capacity in human plasma using a 96-well microplte. J Clin Biochem Nutr 2009;44:46-51.

https://doi.org/10.3164/jcbn.08-162.

18. Re R, Pellegrini N, Proteggente A, Pannala A, Yang M, Rice-Evans C. Antioxidant activity applying an improved ABTS radical cation decolorization assay. Free Radic Biol Med 1999;26:1231-7.

https://doi.org/10.1016/s0891-5849(98)00315-3

19. Kamal AAM, Gomaa A, Khafif ME, Hammad AS. Plasma lipid peroxides among workers exposed to silica or asbestos dusts. Environ Res 1989;49:173-80.

https://doi.org/10.1016/s0013-9351(89)80062-3.

20. Cawthon RM, Smith KR, O'Brien E, Sivatchenko A, Kerber RA. Association between telomere length in blood and mortality in people aged 60 years or older. Lancet 2003;361:393-5.

https://doi.org/10.1016/s0140-6736(03)12384-7.

21. Woo J, Tang NL, Suen E, Leung JC, Leung PC. Telomeres and frailty. Mech Ageing Dev 2008;129:642-8.

https://doi.org/10.1016/j.mad.2008.08.003.
22. Saum KU, Dieffenbach AK, Müezzinler A, Müller $H$, Holleczek $B$, Stegmaier $\mathrm{C}$, et al. Frailty and telomere length: Cross-sectional analysis in 3537 older adults from the ESTHER cohort. Exp Gerontol 2014;58:250-5. https://doi.org/10.1016/j.exger.2014.08.009.

23. Yu R, Tang N, Leung J, Woo J. Telomere length is not associated with frailty in older Chinese elderly: Cross-sectional and longitudinal analysis. Mech Ageing Dev 2015;152:74-9.

https://doi.org/10.1016/j.mad.2015.10.002

24. Zhou J, Wang J, Shen Y, Yang Y, Huang P, Chen S, et al. The association between telomere length and frailty: A systematic review and meta-analysis. Exp Gerontol 2018;106:16-20.

https://doi.org/10.1016/j.exger.2018.02.030.

25. Volpato S, Leveille SG, Corti MC, Harris TB, Guralnik JM. The value of serum albumin and high-density lipoprotein cholesterol in defining mortality risk in older persons with low serum cholesterol. J Am Geriatr Soc 2001;49:1142-7. https://doi.org/10.1046/j.1532-5415.2001.49229.x.

26. Schalk BWM, Visser M, Deeg DJH, Bouter LM. Lower levels of serum albumin and total cholesterol and future decline in functional performance in older persons: The longitudinal aging study Amsterdam. Age Ageing 2004;33:266-72. https://doi.org/10.1093/ageing/afh073.

27. Bartali B. Poor nutrition with aging: An "oxidatively stressing" condition. Curr Nutr Food Sci 2007;3:216-21.

https://doi.org/10.2174/157340107781369170.

28. Slee A, Birch D, Stokoe D. The relationship between malnutrition risk and clinical outcomes in a cohort of frail older hospital patients. Clin Nutr ESPEN 2016;15:57-62.

https://doi.org/10.1016/j.clnesp.2016.06.002.

29. Wu IC, Shiesh SC, Kuo PH, Lin XZ. High oxidative stress is correlated with frailty in elderly Chinese. J Am Geriatr Soc 2009;57:1666-71.

30. Shamsi KS, Pierce A, Ashton AS, Halade DG, Richardson A, Espinoza SE. Proteomic screening of glycoproteins in human plasma for frailty biomarkers. J Gerontol A Biol Sci Med Sci 2012;67:853-64.

https://doi.org/10.1093/gerona/glr224.

31. Ramaty E, Maor E, Peltz-Sinvani N, Brom A, Grinfeld A, Kivity S, Segev S, et al. Low ALT blood levels predict long-term all-cause mortality among adults. A historical prospective cohort study. Eur J Intern Med 2014;25:919-21. https://doi.org/10.1016/j.ejim.2014.10.019.

32. Dasgupta A, Klein K. Methods for Measuring Oxidative Stress in the Laboratory. Antioxidants in Food, Vitamins and Supplements. Ch. 2. San Diego: Elsevier; 2014. p. 19-40. https://doi.org/10.1016/b978-0-12-405872-9.00002-1.

33. Fougère $B$, Vellas $B$, Van Kan GA, Cesari M. Identification of biological markers for better characterization of older subjects with physical frailty and sarcopenia. Transl Neurosci 2015;6:103-10.

https://doi.org/10.1515/tnsci-2015-0009.

34. Inglés M, Gambini J, Carnicero JA, García-García FJ, Rodríguez-Mañas L, Olaso-González $\mathrm{G}$, et al. Oxidative stress is related to frailty, not to age or sex, in a geriatric population: Lipid and protein oxidation as biomarkers of frailty. J Am Geriatr Soc 2014;62:1324-8.

https://doi.org/10.1111/jgs.12876.

35. Soysal P, Isik AT, Carvalho AF, Fernandes BS, Solmi M, Scofield P, et al. Oxidative stress and frailty: A systematic review and synthesis of the best evidence. Maturitas 2017;99:66-72. https://doi.org/10.1016/j.maturitas.2017.01.006.

36. Collerton J, Martin-Ruiz C, Davies K, Hilkens CM, Isaacs J, Kolenda C et al. Frailty and the role of inflammation, immunosenescence and cellular ageing in the very old: Cross-sectional findings from the Newcastle $85+$ study. Mech Ageing Dev 2012;133:456-66. https://doi.org/10.1016/j.mad.2012.05.005 